\section{SYLLABUS DEL CURSO: HISTORIA SOCIAL DE LA SALUD PÚBLICA EN AMÉRICA LATINA*}

\section{COURSE SYLLABUS : THE SOCIAL HISTORY OF PUBLYC HEALTH IN LATIN AMERICA}

\author{
Marcos Cueto y Anne-Emanuelle Birn**
}

\section{Introducción}

Las personas, profesiones e instituciones desarrollan una imagen de su propio pasado que les sirve para perfilar una identidad, cohesionar a sus miembros y reflexionar sobre su futuro. En el caso de la salud pública contemporánea, la necesidạd de reformular esta imagen es cada día mảs urǵente por la sensación de crisis, cuestionamiento, y reorientación que experimenta el sector. Esta reformulación debe evitar caer en las narraciones anécdóticas, enciclopédicas y celebratorias que impiden reflexionar con claridad y objetividad sobre el pasado y las opciones de la salud pública. Por ello, es necesario construir una nueva imagen de la salud pública a partir del examen del conocimiento e interpretación que brindan los historiadores profesionales sobre la salud pública en Europa, Estados Unidos y América Latina y el Caribe. La organización de cursos sobre la historia social de la salud pública debe contribuir a lograr este objetivo y, en este sentido, llenar las lagunas en la formación de los salubristas y ampliar el panorama de temas de interés de los historiadores.

La elaboración de este syllabus tiene varios motivos y está inspirada en diversos ejemplos. Algunos de esos ejemplos fueron producidos hace algunos años por investigadores, que tratando de hacer de la historia social de la ciencia un campo de contornos definidos, publicaron artículos con cursos y bibliografías ideales que sirvieron como lecturas para investigadores y en el diseño de cursos para alumnos universitarios. ${ }^{1}$ Uno de los objetivos de este syllabus es tratar de reproducir esta experiencia de la historia de la ciencia para la historia de la salud pública en América Latina.

Otra motivación de este syllabus es contribuir al interés mostrado en los últimos años por parte de investigadores y docentes de la región en la historia de la salud. Este interés se ha visto enfrentado muchas veces con la escaza disponibilidad de recursos bibliográficos, educacionales, financieros, y de conservación de documentos. Asimismo, con la poca comunicación y colaboración entre investigadores y entre ellos y las instituciones.

\footnotetext{
* Este trabajo apareció en Salud, cullura y sociedad en América Lalina: nuevas perspeclivas bistöricas, M. Cueto (ed.), Lima, Instituto de Estudios Peruanos, 1996, y es parte del Programa de Investigación de la División de Salud y Desarrollo de la Organización Panamericana de la Salud. Su coordenador, Alberto Pellegrini Filho, lanzó en 1994 una iniciativa que busca el desarrollo de la investigación histórica en salud en América Latina con los seguientes componentes complementarios: a) una red electrónica de investigadores de historia de salud pública en la región; b) el montaje de la Bibliografia da História da Saúde Publica na América Latina e Caribe (Hisa), coordenada por la Casa de Oswaldo Cruz, com el apoyo del Centro Latino-Americano e do Caribe de Informação em Ciências da Saúde (Bireme), en periodo de prueba, la qual deverá estar materializada al comienzo del próximo año; c) un concurso de investigación bajo el tema 'Reforma sanitaria en perspectiva histórica en América Latina y el Caribe; y d) un syllabus de un curso de historia social de la salud pública. La biblioteca de la Casa de Oswaldo Cruz tendrá copia de los artículos y documentos incluídos en la bibliografía. Ilistória, Ciências, Sauide Manguinhos reproduce la totalidad de esta propuesta para incentivar la realización de nuevos cursos de historia de la salud pública y también para fomentar el debate sobre el conteniclo de cursos ya existentes (Nota del edilor). ** Historiador, director de investigaciones del Instituto de Estudios Peruanos y historiadora, consultora de la OPAS. Instituto de Estudios Peruanos

Horacio Urteaga 694 Lima

e-mail: mcueto@iep.pe

1 Por ejemplo, Brian Easlea, 'An introduction to the history and social studies of science: a seminar course for firstyear science students', Science Sudies, 3: 185-209, 1973; y Steven Shapin, 'Historia social de la ciencia', Mathesis: Revista de Divulgación e Información en Filosofía e Historia de las Matemáticas, 3: 295-320, 1987.
} 
Aunque hace algunos años que se ha reconocido la importancia de la perspectiva de la historia social de la ciencia en América Latina, representado en parte por los miembros de la Sociedad Latinoamericana de la Historia de la Ciencia y de la Tecnología creada en 1982, por la aparición de la revista Quipu en 1984, y por el volumen de trabajos publicados en este campo en la última década, el campo más específico de la historia social de la salud pública en América Latina y el Caribe se encuentra actualmente en una fase embrionaria de desarrollo. ${ }^{2}$

Sin duda, el estado incipiente del campo de historia de la salud pública en América Latina se refleja también en el estado de la enseñanza de esta materia. Para responder a las inquietudes relacionadas directamente a la enseñanza de la historia de la salud pública, hicimos una revisión de syllabus que han sido utilizado para cursos de historia social, historia de la ciencia, políticas de salud, e historia de la medicina en las universidades de la región. También se consultaron extensivamente la Bibliografía de Historia de Salud Pública que desarrolló uno de los autores de este trabajo (Anne-Emanuelle Birn) bajo el auspicio de la OPS (y que ahora tiene sede en la Casa Oswaldo Cruz en Rio de Janeiro). A través de este trabajo se ha tratado de identificar la literatura que nosotros y diversos autores consideramos más significativa y apropiada para la historia social de la salud pública en la región.

La revisión de los cursos en América Latina, junto con nuestras conversaciones con docentes e investigadores, de la región indican algunas deficiencias y lagunas importantes. En primer lugar, existe un problema de escasez. Es decir, la historia de la salud pública está tocada en cursos de la historia de la ciencia pero se dedica a ella solo algunas sesiones. Asimismo, muchos cursos de historia de la medicina tratan de la salud pública como subspecialidad de medicina. Finalmente, la mayoría de los cursos no incorporan el enfoque de la historia social, perdiendo así la importancia fundamental de las interacciones entre la salud pública y la socieclad en distintos períodos históricos.

Asimismo, se ha notado que varios cursos presentan con gran detalle la historia de la salud pública en Europa y Norteamérica y asumen que la transmisión de estos desarrollos como automática y sin problemas, sin tratar las cuestiones fundamentales de la recepción, transformación, y resistencia locales a las icleas y prácticas foraneas. Finalmente, se nota que, con escasas excepciones, hay poca interacción entre los historiadores y los salubristas que investigan, enseñan, y estuclian la historia de la salud pública u otros aspectos relacionados con ciencias sociales y salud. Con esta falta de interacción se pierden, por los dos lados, informaciones, perspectivas, estímulos y recursos valiosos. La comunicación entre investigadores y salubristas puede ser tan mala que nisiquiera un grupo conoce la literatura del otro.

Tenienclo en cuenta la persistencia del modelo tradicional de hacer historia de la medicina uno de los puntos de partida de este trabajo son los supuestos en que se basa la historia social. Las nuevas perspectivas sociales en el campo de la historia surgen de una tendencia iniciada en los años 1930 en Francia por la escuela Annales, la culal otorgó prioridad a nuevas dimensiones del estudio del pasado con el objetivo de recapturar el pasado de los sectores populares y no solo el de las élites políticas, religiosas, y culturales. Asimismo, esta tendencia subrayó la importancia en la búsqueda de nuevos tipos de fuentes de caracter primario. Hasta hace pocos años, la historia de la salud pública en América Latina por lo general no había incorporado este enfoque de historia social.

\footnotetext{
2 Algunos han tratado de la enseñanza de la historia de la ciencia o de la medicina pero la enseñanza de la historia de la salud ha recibido poca atención. En 1994 se inició, en Rio de Janeiro, una revista dedicada en parte a la historia de la salud: IIistória, Ciências, Satide-Manguinhos. Véase Tomás Brody, 'La historia de Ia ciencia en la enseñanza'; Emilio Quevedo, 'Papel de la historia de las ciencias en Ia integración curricular de la Escuela Colombiana de Medicina'; Shozo Motoyama el al., 'História da ciência e o seu ensino na Universidacle cle São Paulo', que aparecieron en Quipu, 1: 195-204; 223-44 y 245-52, 1984; y Mario Hernández y Emilio Quevedo, 'Historia, ciencias sociales y educación médica', Educación Médica y Salud, 26: 35-50, 1992.
} 
Teniendo en cuenta lo anterior, y como perspectiva general, pensamos que el reto en el campo de la historia de la salud pública ha sido y es el de superar la tendencia a enseñar y estudiar de una manera descriptiva el pasado descle la perspectiva de los grandes personajes (generalmente hombres) y la justificación de sus éxitos personales e institucionales. Es importante destacar que esta tendencia tradicional ocurrió porque a menudo los docentes y autores de los trabajos de historia de la salud han sido médicos y salubristas distinguidos que se convirtieron en historiadores aficionados. Aunque este tipo de trabajo ofrece datos valiosos, la historia celebratoria, descriptiva, y narrativa no siempre ha permitido el análisis profundo y crítico de los problemas centrales en el desarrollo de la salud pública.

Con el surgimiento de la historia social y del enfoque social de la salud pública, la historia de la salud pública ha incorporado en los últimos años como objeto de estudio y de enseñanza una serie de nuevos temas: el impacto de la salud pública en el trabajo, en las mujeres, y en grupos minoritarios; el papel sanitario de enfermeras y parteras empíricas; las tendencias de mortalidad y morbilidad por clase social; la percepción y la contextualización social de la enfermedad; la relación entre la medicina y la salud pública; la ideología política y la salud; la enseñanza de la salud pública; la medicina social y el papel social y político del hospital y el impacto de la teoría del germen de la enfermedad; el imperialismo, la medicina tropical, y las campañas sanitarias; el control de enfermedades transmisibles sexualmente y el control social; el papel del gobierno y de los movimientos populares en la salud pública; los cambios en la conceptualización de la salud y de la enfermedad; y el impacto político, social, y médico de los sistemas sanitarios. ${ }^{3}$ El objetivo de este syllabus es de aplicar el nuevo enfoque social al estudio de la historia de la salud pública en América Latina y el Caribe manteniendo la descripción y la interpretación de la importante historia de las instituciones de salud en la región.

Como curso de introducción este syllabus pretende promover algunos de los temas sobresalientes ya mencionados con la idea de incorporar la nueva literatura especializada. Este curso se orienta alrededor de los orígenes, la evolución y la organización de la salud pública, de las instituciones principales encargadas de los servicios de salud, de las interacciones de la salud con las dimensiones sociales, políticas, y culturales, de la ideología de la salud y sus influencias en campos no médicos, y del desarrollo de la cooperación internacional. No estamos tan interesados en la narración anecdótica de los médicos o las terapias que utilizaron. Esperamos que este curso ayudará a aumentar el nivel de reflexión sobre la salud en el presente y los cambios que está viviendo actualmente. Reconocemos el reto de presentar un curso introductorio de la historia de la salud pública a un grupo heterogéneo de estudiantes $e$ investigadores de diferentes nacionalidades pero también pensamos que esta heterogeneidad contribuirá al reconocimiento de la relevancia de la historia para la elaboración de políticas en la actualidad. ${ }^{4}$

Este syllabus no es un modelo rígido sino quiere contribuir a la construcción de cursos que atiendan a los diferentes intereses regionales, profesionales, o intelectuales de los investigadores y de las distintas instituciones de los países. El objetivo de los temas que trata este syllabus es el de describir y analizar la historia social de la salud pública poniendo énfasis en los hechos y corrientes ocurridos durante los siglos diecinueve y veinte. Se prestará especial atención a las principales

3 Al respecto, vêase Charles Rosenberg, 'Introduction. Why care about the history of medicine?', en Explaining epidemics and other studies in the hislory of medicine, Cambridge, Cambridge University Press, 1992, pp, 1-6; y Elizabeth Fee, 'Public health, past and present: a shared social vision', en George Rosen, $A$ hislory of public heallh, Baltimore, Johns Hopkins University Press, 1993, pp. ix-Ixvii.

4 Para las conexiones entre la historia y el análisis de las políticas, Edward D. Berkowitz, 'Public history, academic history, and policy analysis: a case study with commentary', The Public llisiorian, 10: 43-63, 1988; y Peter N. Stearns y Joel A. Tarr, 'Straightening the policy history tree', The Public Itistorian, 15: 63-7, 1993. 
corrientes salubristas de Europa, los Estados Unidos, y América Latina y a los contextos políticos, sociales y culturales en que estas corrientes se desarrollaron. El curso examinará el origen y la evolución del cuidado de la salud ofreciendo una mejor perspectiva para comparar y comprender los problemas actuales que atraviesa la salud pública en América Latina.

Este es un curso introductorio que está diseñado para ser dirigido a investigadores y estudiantes universitarios. Aunque gran parte de nuestra preocupación ha sido el adaptarlo a las posibilidades y necesidades de las escuelas de salud pública de América Latina, es un curso que también puede ser orientado para estudiantes graduados de historia, de otras ramas de las ciencias sociales y a un público general. Está dirigido a brindar una formación mas completa y coherente, estimular la capacidad crítica y comparativa en la región, y promover la investigación entre los futuros salubristas. Aunque no es un seminario de temas de investigación, las lecturas ofrecen un punto de particla necesario para quien quiera realizar una investigación histórica del pasado de la salud. Una aclaración final, hay temas importantes que no han sido incluidos en toda su extensión (como la medicina precolombina y colonial) ó que a pesar de su interés no han sido aún suficientemente investigados (como el desarrollo de la enfermería en Latinoamérica).

El curso está organizado en once sesiones. Cada punto del contenido se refiere a una de las sesiones. Un cuadro resumen muestra el orden de las sesiones, y su interrelación. La secuencia cronológica de las sesiones se debe a dos motivos: primero, como curso introductorio es importante construir un conocimiento de largo plazo, pra entender mejor las tendencias a través del tiempo. Por otro lado un campo como la salud pública se construye a través de paradigmas, bloques de conocimiento, y debates sociales que tienen una relación muy estrecha a momentos políticos particulares. Sin embargo, existen muchas conexiones entre las varias lecturas, y se espera que los docentes y estudiantes explorarán las comparaciones entre épocas diferentes. Aunque la mayoría de las 'lecturas requeridas' son de textos en español se han asignado algunas en inglés y portugués.
Hemos tratado de seleccionar como lecturas requeridas aquellas que permitan la comprensión global de un tema o que sirvan para iniciar una discusión en clase. Muchas veces nos hemos sentido limitados por la falta de estudios históricos sobre ciertos temas, por lo que algunos temas importantes - de la historia reciente - quedan sin tratar como la historia de la medicina social iniciada en los años setenta, la cambiante relación entre la salud y el medio ambiente y el desarrollo de la planificación y de la investigación en salud. Dependiendo de cada país, las lecturas pueden ser adaptadas. Las 'lecturas complementarias' pueden servir de guía de consulta tanto a un profesor que dictal el curso por primera vez como a un estudiante que quiera profundizar su conocimiento sobre algún tema. Por ejemplo, en algunas secciones pueden incluirse algunos textos que ilustren lo que ocurrió en la historia de la salud pública del país donde se dicta el curso. Finalmente, queremos subrayar que este es una propuesta abierta a la discusión y que pueden haber reestructuraciones, lecturas y sesiones que serán bienvenidas por los autores de este trabajo.

\section{Cuadro Resumen de las 11 sesiones del Curso de Historia Social de la Salud Pública en América Latina}

1. Historia y Salud. Introducción al Curso.

2. El Modelo Hipocrático-Galénico y las Instituciones Coloniales.

3. El Surgimiento del Estado y la Salud Pública en Europa.

4. Los Inicios de la Medicina y de la Salud Pública en América Latina.

5. La Salud Pública en la Europa del Siglo XIX.

6. La Salud Pública en América Latina durante el Siglo XIX.

7. La Bacteriología y la Teoría del Germen de la Enfermedad.

8. El Impacto de la Bacteriología en América Latina.

9. El Modelo y la Influencia Norteamericana.

10. La Institucionalización de la Salud Pública en América Latina.

11. La Salud Pública en América Latina desde 1950. 


\section{Historia y Salud. Introducción al Curso}

Existen dos objetivos para esta primera sesión. En primer lugar, discutir el valor de la perspectiva histórica en la comprensión de los problemas de la salud. Esta discusión implica presentar las ventajas de una visión de larga duración sobre los origenes, el desarrollo, las innovaciones y las rupturas de la salud pública. El segundo objetivo de esta sesión es diferenciar la historia tradicional, descriptiva ó positivista de la historia social, analítica y cuestionadora de los problemas contemporáneos. Asimismo, puede explicarse las coincidencias y diferencias entre la historia social, la historia de la ciencia, la historia de la medicina y la historia de la salud pública y ofrecerse un panorama general de los estudios recientes de la historia de la medicina y de la salud.

\section{Lecturas requeridas}

Arrizabalaga, Jon 1992

Rosenberg, Charles 1992

Saldaña, Juan José (ed.) 1986

\begin{abstract}
'Nuevas tendencias en la historia de la enfermedad: a propósito del constructivismo social'. Arbor, 143: 147-65.
\end{abstract}

\begin{abstract}
'Framing disease: illness, society and history'. En Charles Rosenberg y Janet Golden (eds.), Framing disease: studies in cultural bistory. New Brunswick, Rutgers University Press, pp. xiii-xxvi.

'Marcos conceptuales de la historia de las ciencias en Latinoamérica: positivismo y economicismo'. En El perfil de la ciencia en América. México, Cuadernos de Quipu, pp. 57-81.
\end{abstract}

\section{Lecturas complementarias}

Fee, Elizabeth

1993

Lafuente, Antonio y

Ortega, Maria Luisa

1992

Portocarrero, Vera 1993
'Public health, past and present: a shared social vision'. En George Rosen, A bistory of public health. Baltimore, Johns Hopkins

University Press, pp. ix-lxvii.

'Modelos de mundialización de la ciencia'.

Arbor, 143: 93-117.

'Contribuições metodológicas para a história e filosofia das ciências biomédicas: as análises de Salomon-Bayet, Michel Foucault, Francois Jacob e Georges Canguilhem'. Estudos de História e Satide, 6. Rio de Janeiro, Casa de Oswaldo Cruz. (esgotado)

\section{El Modelo Hipocrático-Galénico y las Instituciones Coloniales}

Esta sesión incluirá un análisis del contenido y la persistencia del modelo hipocrático-galénico. De especial interés es el debate sobre el origen de la Teoría de los Humores y de la dualidad frío-caliente y la relación de esta dualidad con la medicina tradicional. Según algunos autores como Foster, esta dualidad es básicamente una importación europea que se difundió popularmente durante el período colonial. Por su lado, autores como Bastien consideran que la concepción humoral manejada por las poblaciones indígenas es de origen precolombino. Asimismo, se analizarán el rol asistencial de las principales instituciones médicas, ordenes religiosas y hospitales que surgieron entre los siglos XVI y XVIII en América Latina.

\section{Lecturas requeridas}

Alchon, Suzanne Austin
'Tradiciones médicas nativas y resistencia en el Ecuador colonial'. En Marcos Cueto (ed.), Saberes andinos: ciencia y tecnología en Bolivia, Ecuador y Perí. Lima, Instituto de Estudios Peruanos, pp. 15-36. 


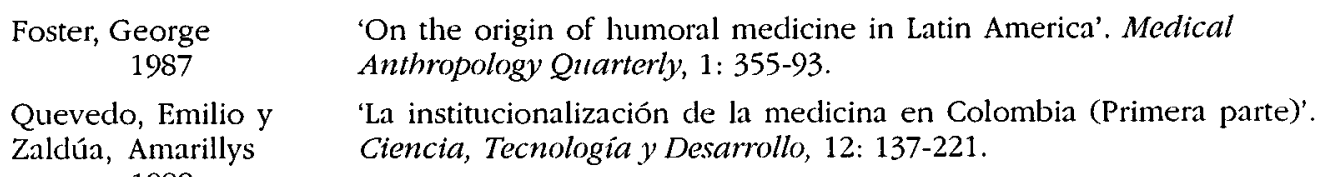

\section{Lecturas complementarias}

Bastien, Joseph 'Differences between Kallawaya-Andean and Greek-European humoral 1989 theory'. Social Science and Medicine, 28: 45-51.

Cooper, Donald B. Epidemic disease in Mexico City, 1716-1813: an administrative, social, and 1965 medical study. Austin, University of Texas Press.

Fisher, Lawrence E. Colonial madness: mental health in the barbadian social order. New 1985 Brunswick, Rutgers University Press.

Guerra, Francisco 'La medicina popular en Hispano América y Filipinas'. Asclepio, 25: 323-30. 1973

Risse, Guenter B. 'Medicine in New Spain'. En Ronald Numbers (ed.), Medicine in the New 1987 World, New Spain, New France and New England. Knoxville, The University of Tennessee Press, pp. 12-63.

Silva, René

1992

Las epidemias de vinuela de 1782 y 1802 en la Nueva Granada: contribución a un análisis histórico de los procesos de apropiación de modelos culturales, Cati, Universidad del Valle.

Vázquez, J. Carlos Aguado y Barboza, Xóchitl Martínez 'El concepto de caridad de la atención médica en la Nueva España'. En y Gonzalo Aguirre Beltrán y Roberto Moreno de los Arcos (eds.), Historia general de la medicina en México: medicina novobispana, siglo XVI. 1990 México, Unam, vol. II, pp. 272-8.

\section{El Surgimiento del Estado y la Salud Pública en Europa}

Esta sesión se iniciará con un debate de la influencia del mercantilismo y la ilustración en las ideas sobre la población y la salud pública. Un aspecto contextual importante para entender este debate es la importancia atribuida a la salud pública y a la medicina para consolidar el poder de los emergentes estados nacionales europeos. De especial interés es el análisis de medidas promovidas por el Estado que tuvieron impacto en el control social, el desarrollo de las profesiones y la organización del espacio urbano como la creación de la policía médica, la reforma de la educación médica que acabó con la distinción entre médicos y cirrijanos, la secularización de los hospitales que estaban en manos de las ordenes religiosas, la construcción de cementerios extramuros y las diversas disposiciones de saneamiento urbano. Finalmente, puede iniciarse en esta sección una discusión sobre el impacto de la medicina y de la salud pública en la reducción de la mortalidad europea.

\section{Lecturas requeridas \\ Mckeown, Thomas 1982 \\ Rosen,George 1985}

Rosen,George

'Los conceptos de salud y enfermedad'. En Thomas McKeown, El papel de la medicina: sueño, espejismo o némesis. México, Siglo XXI, pp. 15-23.

'El mercantilismo y la política para la salud en el pensamiento francés del siglo XVIII'. En George Rosen, De la policía médica a la medicina social. México, Siglo XXI, pp. 228-53.

‘Que es la medicina social? Un análisis genético del concepto?'. En Rosen, De la policía médica, pp. 77-113. 


\section{Lecturas complementarias}

Foucault, Michel 1986

Riley, James C. 1987

Szreter, Simon 1988
El nacimiento de la clínica: una arqueología de la mirada médica. México, Siglo XXI.

The eighteenth century campaign to avoid disease. New York, St. Martin's Press.

'The importance of social intervention in Britain's mortality decline, 18501914: a reinterpretation of the role of public health'. Social History of Medicine, 1: 1-37.

\section{Los Inicios de la Medicina y de la Salud Pública en América Latina}

Esta sección se concentrará en la influencia de la ilustración europea en la medicina de la región, con especial atención al rol de las reformas borbónicas, entre fines del siglo XVIII y comienzos del siglo XIX. Estas reformas estuvieron dirigidas a reafirmar el poder de la corona española sobre sus posesiones americanas y alentaron la formación de una élite intelectual que incorporó a sus preocupaciones los problemas sanitarios. Se analizará la creación de cátedras de anatomía y escuelas de medicina, y la importancia de las expediciones botánicas que estudiaron la quina y la expedición de la vacuna contra la viruela.

\section{Lecturas requeridas}

Clement, Jean $\quad$ 'El nacimiento de la higiene urbana en la América Española del siglo XVIII'.

Pierre Revista de Indias, 49: 77-94.

1983

Lafuente, Antonio y 'Las profesiones sanitarias tras su identidad en la ilustración española'. En

Sarmiento, Javier Historia social de la ciencia, sabios, médicos y boticarios. Bogotá,

Puerto Universidad Nacional de Colombia, pp. 41-59.

1989

Quevedo, Emilio y 'Antecedentes de las reformas médicas del siglo XVIII y el XIX en el Nuevo

Zaldúa, Amarillys Reino de Granada. Una polémica entre médicos y cirujanos'. Quipu, 3: 311-34. 1986

Lecturas complementarias

Arquiola, Elvira 'La expedición de Balmis y la clifusión de la vacuna'. En Alejandro Diez Torre 1993 (ed.), La ciencia española en ultramar. Madrid, Ed. Doce Calles, pp. 249-54.

Crosby, Alfred W. Jr. The Columbian exchange: biological and cultural consequences of 1492. 1972 Westport, Greenwood Press.

Estrella, Eduardo 'Expediciones botánicas'. En Jose L. Peset y Antonio Lafuente, Carlos III y la 1989 ciencia de la ilustración. Madrid, Alianza Universidad, pp. 331-52.

Lafuente, Antonio y 'Ciencia colonial y roles profesionales en la América Española

Catala, José Sala del siglo XVIII’. Quipu 6: 387-403. 1989

\section{La Salud Pública en la Europa del Siglo XIX}

Esta sesión discutirá la emergencia del rol del salubrista y de la preocupación por el medio ambiente durante el siglo XIX en Inglaterra, Alemania, y Francia. Esta discusión implica el contraste, tensión y puntos en común entre las ideas contagionistas y anticontagionistas (ó miasmáticas). Un aspecto importante es relacionar las implicaciones para el comercio de cada uno de estas corrientes. Es importante superar la asociación tradicional establecida entre los contagionistas y la epidemiología moderna para reevaluar la contribución de ambas 
corrientes a la preocupación por los factores sociales y ambientales que causaban la enfermedad. Finalmente, se presentarán las características generales del modelo de medicina e higiene francesas dominante durante la primera mitad del siglo XIX. Entre los factores sociales y políticos más importantes a ser tomados en cuenta para ubicar la obra de personajes como Chadwick, Snow, Villermé, y Virchow, se encuentran la revolución inclustrial, el crecimiento de la clase obrera, el trabajo de mujeres y niños, y la urbanización sin infraestructura sanitaria.

\section{Lecturas requeridas}

Ackerknecht, Erwin 'Anticontagionism between 1821 and 1867'. 1948. Bulletin of the History of Medicine, 22: 562-93.

García, Juan César '1848: nacimiento de la medicina social'. En J. C. García, Pensamiento social en 1994 salud en América Latina. Washington, OPS/Interamericana McGraw-Hill, pp. 144-9.

Rosen, George 'Industrialism and the Sanitary Movement (1830-1875)'. En Rosen, A history of public health, pp. 168-269.

\section{Lecturas complementarias}

Berge, Ann La and French medical culture in the $19^{\text {th }}$ century. London, Clio Medica.

Feingold, M. (eds.) 1993

Coleman, William Death is a social disease, public health and political economy in early industrial 1982 France, Madison, University of Wisconsin Press.

Evans, Richard J. Death in Hamburg, society and politics in the cholera years, 1830-1910. 1987 Oxford, Claredon Press.

Rosenberg, Charles The cholera years, 1832, 1849, and 1866. Chicago, 1962 University of Chicago Press.

\section{La Salud Pública en América Latina durante el Siglo XIX}

Esta sección discutirá el desarrollo de la medicina y de la sanidad Latinoamericana durante el siglo XIX. Desde comienzos de ese siglo, la sucesión de epidemias que interrumpian el desarrollo de las economías de exportación, la modernización y estabilización de los estaclos luego de las guerras civiles y la llegada de inmigrantes europeos, entre otros factores, contribuyeron a la formación de juntas de sanidad de emergencia, a la adscripción de respon- sabilidades sanitarias a las municipalidades y a otras agencias de gobierno local, a la organización de medidas de saneamiento urbano y a la imitación de modelos de medicina y salud europeos. La discusión incluirá el papel que jugó la influencia europea en la organización de la enseñanza médica profesional y en el diseño de los grandes hospitales. El caso de Brasil ilustra una dimensión cultural importante de la salud, es decir como la discusión y el control sanitario influenciaron en la percepción sobre las diferencias de razas que existían en los ambientes urbanos.

\section{Lecturas requeridas}

Armus, Diego 1984

Azeredo, Paulo de Roberto 1978
'Enfermedad, ambiente urbano e higiene social: Rosario entre fines del siglo XIX y comienzos del XX'. En D. Armus (ed.), Sectores populares y vida urbana. Buenos Aires, Clacso, pp. 37-66.

Clase social e saúde na cidade do Rio de Janeiro: primera metade do século XIX'. Revista do Museu Paulista, 25: 129-65. 
Chalhoub, Sidney 1993

Pruna, Pedro 1991
'The politics of disease control: yellow fever and race in nineteenth century Brazil'. Joumal of Latin American Studies, 25: 441-64.

'La vacunación homeopática contra la fiebre amarilla en la Habana, 1855'. Asclepio, 43: 59-68.

\section{Lecturas complementarias}

Anderson, Robin L. $\quad$ 'Public health and public healthiness, São Paulo, Brazil, 1876-1893'. Journal 1986 of the History of Medicine and Allied Sciences, 41:293-307.

Asúa, Miguel de 1986.

'Influencia de la Facultad de Medicina de París sobre la de Buenos Aires'. Quipu, 3: 79-90.

Escudé, C. A. 1989

'Health in Buenos Aires in the second half of the nineteenth century'. En D. C. Platt (ed.), Social welfare, 1850-1950: Australia, Argentina and Canada compared. Basingstoke, Macmillan, pp. 60-70.

\section{La Bacteriología y la Teoria del Germen de la Enfermedad}

Esta sesión analizará el impacto de la bacteriología en la medicina y en la salud pública. Un tema a debatirse es si la teoría del germen de la enfermedad contribuyó a una visión unicausal, reduccionista y medicalizada de los problemas sanitarios que opacó las reflexiones sociales y ambientales de algunos sanitaristas clel siglo diecinueve. Asimismo, se analizará como varían las percepciones sobre la enfermedad según el período histórico y como sobreviven concepciones sociales más amplias sobre las enfermedades infecciosas. Se presentarán las características generales de los modelos institucionales creados por Pasteur en París y Koch en Berlin, que fueron posteriormente exportados a Latinoamérica. También se discutirá la interacción entre la teoría del germen y los intereses imperialistas europeos que hicieron surgir una nueva especialidad médica: la medicina tropical. El caso de Inglaterra ilustra con claridad la tensión entre los intereses económicos, sanitarios y científicos que ocurrió en la medicina tropical.

\section{Lecturas requeridas}

Cunningham, Andrew 'La transformación de la Peste: el laboratorio y la identidad de las 1991

Latour, Bruno 1983 enfermedades infecciosas'. Dynamis, 11: 27-72.

'Give me a laboratory and I will raise the world'. En Karin D. Knorr-Cetina y Michael Mulkay (eds.), Science observed, perspectives on the social study of science. London, Sage Publication, pp. 141-70.

Worboys, Michael 1976

'The emergence of tropical medicine: a study in the establishment of a scientific speciality'. En G. Lemaine et al., Perspectives on the emergence of scientific disciplines. The Hague, pp. 75-98.

\section{Lecturas complementarias}

Arnold, David 1988

Leavitt, Judith Walzer 1992

Rosen, George

Weindling, Paul 1992
Imperial medicine and indigenous societies. Manchester, Manchester University Press, pp. 1-26.

'Typhoid Mary' strikes back: bacteriological theory and practice in early twentieth-century public health'. Isis, 83: 608-29.

'The bacteriological era and its aftermath (1875-1950)'. En Rosen, A bistory of public beallh, pp. 270-319.

'Scientific elites and laboratory organization in Paris and Berlin'. En A. Cunningham (ed.), The laboratory revolution in medicine. Cambridge, pp. 170-88. 


\section{El Impacto de la Bacteriología en América Latina}

Esta sesión describirá la llegada de la influencia bacteriológica europea a la región y la creación de institutos de bacteriología en las principales ciudades, los cuales combinaban la investigación de enfermedades nativas con la producción de sueros y vacunas. Como dos estudios de caso importantes pueden discutirse los descubri- mientos del cubano Finlay sobre el vector de la fiebre amarilla y la evolución del Instituto creado por Oswaldo Cruz en Rio de Janeiro. Entre los factores sociales que interactuaron con el desarrollo de la salud pública y que deben ser enfatizados se encuentran la expansión del comercio de exportación, el crecimiento del Estado y las ciudades, y la llegada masiva de inmigrantes europeos a América Latina.

\section{Lecturas requeridas}

Benchimol, Jaime L. 1990

García, Juan César 1981

Stepan, Nancy L. 1978
'Origens e evolução do Instituto Oswaldo Cruz no periodo 1899-1937'.

En Manguinhos, do sonho à vida: a ciência na Belle Époque Rio de Janeiro, Casa de Oswaldo Cruz/Fiocruz, pp. 5-88

'Historia de las instituciones de investigación en salud en América Latina, 1880-1930'. Educación Médica y Salud, 15: 71-88.

'The interplay between socio-economic factors and medical science,yellow fever research in Cuba and the United States'. Social studies of science, 8: 397-424.

\section{Lecturas complementarias}

Benchimol, Jaime L. y Teixeira L. Antonio 1990

Cueto, Marcos 1991

Delaporte, François 1991

Lówy, Ilana 1990

Stepan, Nancy 1981
Cobras, lagartos \& outros bichos. Rio de Janeiro, Fiocruz.

'La ciudad y las ratas: la peste bubónica en Lima y en la costa peruana a comienzos del siglo veinte'. Histórica, 15: 1-26.

The history of yellow fever: an essay on the birth of Tropical Medicine. Cambridge, MIT Press.

'Yellow fever in Rio de Janeiro and the Pasteur Institute Mission (1901-1905): the transfer of science to the periphery'. Medical History, 34: 144-63.

Beginnings of Brazilian science: Oswaldo Cruz, medical research and policy, 1890-1920. New York, Science History Publications.

\section{El Modelo y la Influencia Norteamericanas}

El primer objetivo de esta sección es describir las principales características del modelo de salud norteamericano tomando como puntos de referencia el Informe Flexner de 1910, y la organización de la Escuela de Salud de la Universidad Johns Hopkins. El segundo objetivo es analizar las actividades de la Fundación Rockefeller en América Latina como un medio de difusión de la influencia norteamericana. En este sentido mencionamos como un producto académico de gran impacto y frecuentemente citado en los últimos documentos del sector salud el libro de Elizabeth Fee, Disease and discovery, que identifica las opciones que enfrentó en sus origenes la salud pública contemporánea: la que enfatizaba la ingeniería sanitaria (propuesta en común acuerdo por las universidades de Harvard y MIT); la que priorizaba la participación de las ciencias sociales (seguida en la universidad de Columbia); y la de John Hopkins - cue finalmente predominó con el apoyo de la Fundación Rockefeller - y que ha sido usualmente descrita como biologicista y medicalizada. Las campañas de la Rockefeller contra la fiebre amarilla, la uncinariasis, y la malaria en la región se prestan para una discusión sobre los temas de las motivaciones, prioridades, e impacto de la Fundación. 


\section{Lecturas requeridas}

Agudelo, Saul Franco 1990

Birn, Anne-Emanuelle 1995

Cueto, Marcos 1994
El paludismo en América Latina. Guadalajara, Universidad de Guadalajara, pp. 195-231.

¿El pasado como presagio?: México, la salud pública y la Fundación Rockefeller'. En Leticia Robles Silva y Francisco Javier Mercado (eds.), Memorias del Sexto Congreso Latinoamericano de Medicina Social. Guadalajara, Universidade de Guadalajara, pp 135-55.

'Visions of science and development: The Rockefeller Foundation's Latin American surveys of the 1920's'. En Marcos Cueto (ed.), Missionaries of science, the Rockefeller Foundation and Latin America. Bloomington, Indiana University Press, pp.1-22.

\section{Lecturas complementarias}

Candeias, Martins

Nelly Ferreira 1984

Fee, Elizabeth 1987

Flexner, Abraham 1910

Starr, Paul

Williams, Steven C. 1994

'A história da Faculdade de Saúde Pública da Universidade de São Paulo: 1918-1945'. Revista de Satide Plíblica, 18: 2-60.

Disease and discovery: a history of the Johns Hopkins School of Hygiene and Public Health, 1916-1939. Baltimore, Johns Hopkins University Press.

Medical education in the United States and Canada. New York, Carnegie Foundation for the Advancement of Teaching, Bulletin $\mathrm{n}^{\mathrm{O}} 4$.

'La educación médica y el restablecimiento del control de las ocupaciones', y 'Los límites de la salud pública'. En P. Starr, La transformación social de la medicina, pp. 140-56; 218-37.

'Nationalism and public health: the convergence of Rockefeller Foundation technique and Brazilian federal authority during the time of yellow fever, 1925-1930'. En Marcos Cueto, Missionaries of science. Indiana University Press, pp. 23-51.

\section{La Institucionalización de la Salud Pública en América Latina}

En esta sección debe estudiarse la emergencia del estado benefactor y el surgimiento de los sistemas nacionales de salud. Ello incluye el estudio de los esfuerzos por centralizar los servicios de salud así como la tensión y acomodación entre el sector estatal y otras entidades y actividades dedicadas al cuidado de la salud como las municipalidades, la salud comunitaria, la medicina doméstica, y la medicina tradicional. Asimismo, esta sección debe describir la evolución de las oficinas ó direcciones de sanidad, que generalmente estaban bajo la supervisión de los ministerios del interior, economía, o fomento, y el surgimiento de ministerios de salud autónomos. Esta sesión debe considerar los factores contextuales que contribuyeron a esta evolución prestando particular atención al crecimiento de las profesiones médicas y a las nuevas relaciones que se establecen entre el Estado y la medicina. Finalmente, esta sección discutirá las iniciativas desde abajo que se produjeron en la medicina rural y en las provincias. Esta discusión analizará las condiciones locales que permitieron la emergencia de estas corrientes y los factores sociales y políticas que contribuyeron a la ausencia de vigor y autoridad de estas opciones alternativas.

\section{Lecturas requeridas}

Cueto, Marcos

García, Juan César 1980
'Indigenismo and rural medicine in Peru: the Indian sanitary brigade and Manuel Nuñez Butrón'. Bulletin of the History of Medicine, 65: 22-41.

'La medicina estatal en América Latina, 1880-1930'.

Revista Latinoamericana de Salud, 1: 70-110. 
Santos, Luiz A. de Castro 1993
'A reforma sanitária pelo alto: o pioneirismo paulista no início do século $\mathrm{XX}$ '. Dados, 36: 361-93.

\section{Lecturas complementarias}

Belmartino, S.; Bloch, C.; Persello A. V. y Camino, M., I. 1988

Hochman, Gilberto 1993

Llamas, Héctor

Hernández 1982

Márquez, Patricio

y Joly, Daniel J. 1986

Merhy, Emerson Elias 1992

Zilvetti, Olga Palmero 1981
Corporación médica y poder en salud, Argentina, 1920-1945. Buenos Aires, Organización Panamericana de la Salud, pp. 15-32, 155-200.

'Regulando os efeitos da interpendência sobre as relações entre saúde pública e construção do Estado (Brasil 1910-1930)'.

Estudos Históricos, 11: 40-61.

'Historia de la participación del Estado en las instituciones de atención médica en México'. En Federico Ortiz Quesada (ed.), Vida y muerte del mexicano. México, D.F. Folios Ed., pp. 49-96.

'An historical overview of the ministries of public health and the medical programs of the social security systems in Latin America'. Joumal of Public Health Policy, 7: 378-94.

A satide pública como política: 1 m estudo de formuladores de políticas. São Paulo, Hucitec.

'La seguridad social y la atención de la salud en América Latina'. Revista Méxicana de Ciencias Politicas y Sociales, 27: 181-202.

\section{La Salud Pública en América Latina desde 1950}

Esta sesión discutirá la evolución de la salud pública en la región desde la Segunda Guerra Mundial hasta nuestros días. De especial importancia es el análisis de los factores ideológicos, sociales, y gremiales que permitieron la emergencia y el desarrollo de los seguros sociales, la reformulación del rol de los hospitales y el desarrollo de la enfermería y de la salud pública como una profesión. La discusión sobre el contexto incluirá el papel jugado por los empresarios industriales, los partidos políticos populistas, los médicos, y los sindicatos en la promoción y resistencia de la ampliación de las políticas sociales del estado. Asimismo, como un estudio de caso se discutirá el contenido y el contraste entre los conceptos de control y erradicación de la malaria. Finalmente, se dará especial atención al desarrollo de la medicina social en la región.

\section{Lecturas requeridas}

Agudelo, Saul Franco

Belmartino, S. et al. (eds.)

1991

Castrillón, Maria

Consuelo 1986

Duarte, Everardo Nunes

1991
El paludismo en América Latina. pp. 87-114, 127-70.

'La discusión en torno al seguro'. En Fundamentos históricos de la construcción de relaciones de poder en el sector salud, Argentina, 1940-1960. Buenos Aires, Organización Panamericana de la Salud, pp. 207-33.

'Formación universitaria de enfermeras: Colombia, 1937-1980'. Investigación y educación en enfermeria, 4: 53-72.

'Trayectoria de la medicina social en América Latina: elementos para su configuración'. En Saul Franco et al., Debates en medicina social. Washington, OPS-Alames, pp. 17-137. 
Quevedo E. et al. (eds.) 'La salud pública importada (1948-1957)' y 'La salud y el desarrollo (1958-

1990 1974)'. En La salud en Colombia: un análisis socio-bistórico. Bogotá, Ministerio de Salud, pp. 38-71.

Lecturas complementarias

Duarte, Everardo

Nunes

1994

Illanes, María Angélica

1993

Nájera, José A.

1991

Wainerman, Catalina y Binstock, Georgina (eds.)

1993
'Contribuciones de Juan César García a las ciencias sociales en salud'. En J. C. García, Pensamiento social en salud en América Latina. Washington, OPS/ McGraw-Hill, pp. xiii-xxix.

En el nombre del pueblo, del Estado, y de la ciencia: bistoria social de la salud píblica, Cbile 1880-1973. Santiago, Colectivo de Atención Primaria.

'El paludismo y las actividades de la Organización Mundial de la Salud'. Boletin de la Oficina Sanitaria Panamericana, 111: 131-51.

'Origen histórico de la enfermería en la ciudad de Buenos Aires'. En Ocupación y género: mujeres y varones en enfermería. Buenos Aires, CENEP, pp. 9-29.

\section{OSWAIDO CRUZ: UM MITO DA CIÊNCIA BRASILEIRA}

\section{Nara Brito}

Rio de Janeiro, Fiocruz, 1995.

O momento não poderia ser mais oportuno. Nunca, em nosso país, o interesse do público por uma literatura do tipo biográfico foi tão grande. É claro que tal fenômeno, manifesto no sucesso de vendagem de algumas obras e no patrocínio de editoras ainda durante a elaboração dos livros, não é exclusivamente nacional. Em todo o mundo, este tipo de produção tem envolvido nomes respeitáveis da literatura, do jornalismo e do mundo acadêmico, revelando a diversidade de formas que um tal esforço pode envolver. No caso do Brasil, basta que se recorde a boa acolhida de Olgae, em 1994, de Chatô: o rei do Brasil, ambos de Fernando Morais, além de $O$ anjo pornográfico: a vida de Nelson Rodrigues, de Ruy Castro. A própria polêmica sobre direitos autorais que tumultua a comercialização do último livro deste autor, Estrela solitâria: um brasileiro chamado Garrincha, só virá pôr mais lenha no interesse do grande público leitor. Finalmente, o destaque conseguido, em 1995, pelo Quase memória, quase romance de Carlos Heitor Cony, narrando, entre realidade e ficção, a vida de seu pai jornalista, demonstra a vitalidade do 'gênero bio- gráfico' e as novas e diferenciadas modalidades em que tem sido cultivado.

E quais seriam as razões para tão amplo sucesso, sobretudo se atentarmos que livros de memórias e biografias são um tipo de literatura bem antigo e conhecido, tendo, inclusive, caído em um certo limbo de atenção por parte do público, dos escritores e das editoras, por um bom período de tempo? Acredito que inúmeras poderiam ser as respostas, mas, no que diz respeito a esta resenha, me interessa destacar as transformações que marcaram o campo acadêmico nas áreas da história e das ciências sociais, com a crescente desconfiança e flexibilização dos paradigmas de ordem estrutural, que 'expulsaram' o indivíduo para 'fora' da narrativa. Ou seja, é o retorno do ator, em especial do ator individual, reconhecido como instância de decisão (sempre há escolhas) e como rico fio condutor para o conhecimento de contextos históricos, que fez renascer não só um novo interesse pelo político e pelo cultural, como permitiu um verdadeiro boom na produção de memórias e biografias. Estas não têm mais as características laudatórias tão comuns ao gênero, e mesmo quando o 'biografado' é o próprio autor do texto, guardam um certo cuidado para não cair em exageros comprometedores.

Como se tem observado, esta produção de textos pode compreender romances ou quase romances; memórias ou quase memórias; biogra- 